\title{
Experimental Study on Aerodynamic Characteristics of a Stepped-Nose Obstacle
}

\author{
By Anang CAKRawala and Akira UMEMURA \\ Department of Aerospace Engineering, Graduate School of Engineering, Nagoya University, Nagoya, Japan
}

(Received May 9th, 2003)

\begin{abstract}
Wind tunnel experiments were conducted to obtain a comprehensive understanding of the aerodynamic characteristics of two-dimensional stepped-nose obstacles. In our previous numerical study on the flow around stepped-nose obstacles at a zero angle of attack, the following favorable properties have been found for such step configuration that the flow separating from the front corners reattaches to the leading edges of the side surfaces. (1) The strong vortices trapped in the step region produce the suction forces acting on the step walls to cancel the drag force acting the front surface. (2) The suppression of large-scale flow separation on the obstacle's sides reduces not only the suction force acting on the back surface, but also the lateral force fluctuation to a great degree. (3) The resulting net drag coefficient is much smaller than that of square/rectangular obstacles. In the present experimental study, the drag coefficient of stepped-nosed obstacles with various step height and length, at zero angle of attack, was measured to identify the optimum step configuration for large drag reduction. The effect of attack angle on drag, lift and moment coefficients was examined to gain insight into the static and dynamic stability of stepped-nose obstacles. The effect of step configuration on the Strouhal number was also examined. It was found that the stepped nose brought about static stability to the obstacle with a rather large step length-to-height ratio, but neither static nor dynamic stability was derived for the optimal step configuration with maximum drag reduction.
\end{abstract}

Key Words: Drag Reduction, Stepped-Nose Obstacle, Aerodynamic Instability, Separation Control

\section{Nomenclature}

$C_{D}$ : drag coefficient

$C_{D, \text { un }}$ : uncorrected drag coefficient

$C_{L}:$ lift coefficient

$C_{L, \text { un }}$ : uncorrected lift coefficient

$C_{M}$ : pitching moment coefficient

$C p$ : pressure coefficient

$C p b$ : base pressure coefficient

$C p_{\text {sfw }}$ : pressure coefficient at step frontal wall

$C p_{\text {ssw }}$ : pressure coefficient at step side wall

$f$ : frequency of vortex shedding

$H$ : height of base part of stepped-nose obstacle

$h$ : step height (dimensionless)

$L$ : length of stepped-nose obstacle length $(=W(1+\ell))$

$\ell$ : step length (dimensionless)

$P_{\text {meas }}:$ measured pressure

$P_{\mathrm{s} \text {, ref }}$ : static pressure reference measured at test section inlet

$P_{\mathrm{t}, \text { ref }}$ : total pressure reference measured at test section inlet

Re: Reynolds number

$s$ : distance measured along obstacle's surface

St: Strouhal number

$U_{\infty}$ : uniform stream velocity (test section velocity)

$W$ : width of stepped-nose obstacle

$X$ : coordinate

$Y$ : coordinate

$\alpha$ : angle of attack of uniform stream to obstacle's center line

\section{Introduction}

Flow around blunt bodies has been the subject of intense research in the past, not only through experiment but also by numerical simulation. Typically, the flow around a blunt obstacle consists of shear layer separation, flow reattachment if the obstacle is quite long, and formation of broad wake. A large drag reduction is attainable if flow separation on both sides of the obstacle is suppressed. Interestingly, with the stepped-nose obstacle it is possible to attain an effective drag reduction because the local flow separation at the step regions may produce an effect similar to the rounding-out of sharp front edges. ${ }^{1-3)}$ Hence, this drag reduction technique is useful for many practical applications, e.g. offshore risers, bridge piers, towers, heat exchangers, combustors, and so on. A perspective outlook on blunt body flows, in general, and flow around circular cylinders, in particular, is presented by Koenig and Roshko, ${ }^{4)}$ who investigated the shielding effect of various disks placed coaxially upstream of an axisymmetric body. This shielding effect is closely related to the drag reduction caused by an appropriate stepped-nose obstacle, which is our interest. It is concluded that the drag coefficient decreases for a certain combination of the basic geometric parameters, namely the diameter and gap ratios. For optimum shielding, the stream surface, which separates from the disk, reattaches smoothly onto the front edge of the cylinder. The aerodynamic characteristics of blunt bodies change drastically with width/height ratio. Watanabe ${ }^{1)}$ experimentally showed that notching the perimeter of the flat head of a circular cylinder in step form significantly reduces the drag force acting on the forebody of a circular cylinder whose axis is parallel to the uniform stream. 
In our previous study, ${ }^{2,3)}$ it was found that there is a class of optimum step configuration, for which the net drag force acting on the forebody of a stepped-nose obstacle becomes zero, and the turbulence growth on the side surfaces of a stepped-nose obstacle is suppressed. Optimum step configuration is found when the flow separating from the front corners reaches at the leading edges of the main body's side surfaces. This results in an entrapment of a strong vortex in the step region and the production of a large thrust force in this region. Then, the drag force on the stepped-nose obstacle becomes smaller than the drag on a rectangular obstacle of corresponding size. The net drag is dominated by the back surface pressure, which varies depending on the effect of step corners. It is important to note that the magnitude of suction forces acting at the step regions and on back surface of the obstacle crucially change depending on the sizes of step height and length. Therefore, in order to develop the performance of a stepped-nose obstacle, it is important to have a comprehensive understanding of the flow around the stepped-nose obstacle in various ratios of step length to height with inclined angle of attack. In the present study, the aerodynamic characteristics of stepped-nose obstacles are systematically examined in an experimental approach to identify the most effective stepped-nose configuration, as well as to examine the dynamic stability of the obstacles, i.e., whether the considered stepped-nose obstacle, when subject to small disturbances, could have the tendency to return to a stationary attitude.

\section{Experimental Apparatus and Procedure}

The experiments were conducted in an open circuit low speed wind tunnel at Nagoya University Aerospace Engi-
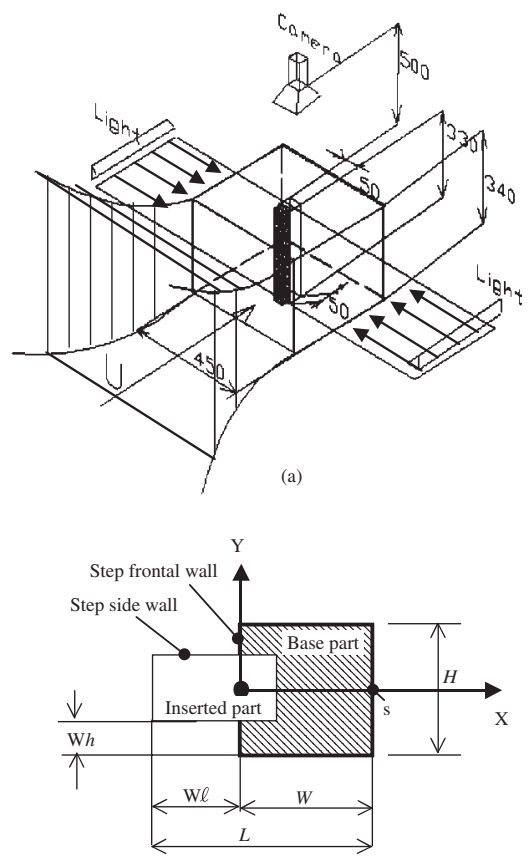

(b)

Fig. 1. Experimental setup and model construction. (a) Wind tunnel sketch, (b) Stepped-nose obstacle sketch. neering Department Propulsion Energy Systems Laboratory. The test section is rectangular with a cross section of $45 \mathrm{~cm} \times 34 \mathrm{~cm}$, as sketched in Fig. 1(a). Figure 1(b) illustrates how to construct the two-dimensional stepped-nose obstacle used in the experiments.

Each stepped-nose obstacle consists of a base part and an inserted part. The inserted part, which has a square cross section, is partially inserted into a casing that constitutes the square form of the base part spanning the top and bottom walls of the test section. The base part has a height $H=$ $50 \mathrm{~mm}$ and width $W=50 \mathrm{~mm}$. The step size was varied by replacing the inserted part. The step height $(W h)$ was determined by the casing thickness, while the step length $(W \ell)$ was determined by the inserted length. Thus, the length of stepped-nose obstacle is $L=W(1+\ell)$. Different inserted parts with various lengths and heights were used in order to obtain various step lengths $(\ell=0.05,0.1,0.2,0.3,0.4)$ and step heights $(h=0.0,0.1,0.2,0.3,0.4)$. The dimensionless step height $h$, equal to 0.5 , means that the stepped-nose obstacle becomes a rectangle of height $H=50 \mathrm{~mm}$ with corresponding lengths $L$. As shown in Fig. 1(a), the two-dimensional stepped-nose obstacle constructed in this way was mounted between the top and bottom walls at the center of the test section, which was located about $5 H$ downstream of the end of the contraction. The solid blockage of the step-
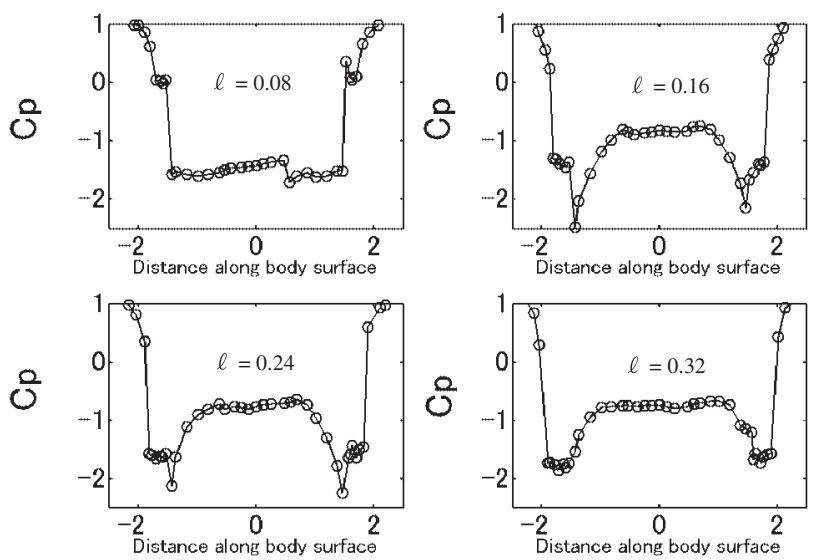

(a) Fixed step height $h=0.16$
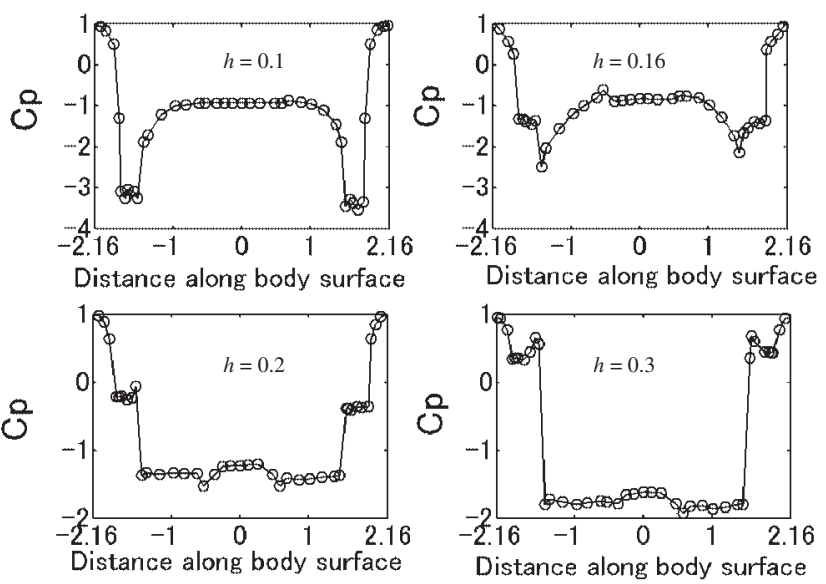

(b) Fixed step length $\ell=0.16$

Fig. 2. Surface pressure distributions 
ped-nose obstacles used in the present experiment was about 10 percent. The experiment was performed in a flow condition which corresponded to a Reynolds number range from $R e=0.5 \times 10^{5}$ to $1.5 \times 10^{5}$ based on the obstacle height $H=50 \mathrm{~mm}$ and a uniform stream velocity $U_{\infty}=10 \sim$ $30 \mathrm{~m} / \mathrm{s}$. It is known that the flow is nominally two-dimensional if the aspect ratio, which is formed by tunnel width, or side plate distance, and height of the step or thickness of the plate, is larger than 10, as described in a paper by Ruderich and Fernholz, ${ }^{5}$ ) who investigated the effect of side plates and aspect ratio on the two-dimensionality of the flow configuration for a forward facing step. In the present experiment, the aspect ratio of tunnel width to step height was 12. For large step height, that was enough to maintain a two-dimensionality for the flow around the stepped-nose obstacle. However, three-dimensional effects were present close to the tunnel walls. Therefore, all measurements were made at the middle section of the stepped-nose obstacle.

The static pressure along the body surface was measured by means of pressure transducers. Pressure orifices with a $0.5 \mathrm{~mm}$ diameter were drilled into both the base part and inserted part, and distributed at an inclination with respect to the uniform stream direction. This was done in order to minimize interference between the pressure orifices. The base part was instrumented with 28 pressure orifices, and the inserted part with 6 to 18 pressure orifices, depending on the step length. To prevent the time lag difference from each orifice measurement, the same length of vinyl tubes were used to connect the transducer with the pressure orifices. For each transducer, we used a scanning device equipped with 48port pressure orifices, where the static pressure, $P_{\mathrm{s} \text {, ref }}$, at the test section inlet was used as a reference. The time-averaged pressure, $P_{\text {mean }}$, was determined within 2 seconds of measurement, during which the sampling frequency was fixed at $5,000 \mathrm{~Hz}$. The pressure was normalized by subtracting the reference pressure and dividing by the uniform stream dynamic pressure $\left(P_{\mathrm{t} \text {, ref }}-P_{\mathrm{s} \text {, ref }}\right)$. The pressure coefficient $C p$ was defined by

$$
C p=\frac{P_{\text {meas }}-P_{\mathrm{s}, \text { ref }}}{P_{\mathrm{t}, \text { ref }}-P_{\mathrm{s} \text {, ref }}}
$$

Drag and lift were calculated by integrating the pressure force acting on the obstacle surface in the direction parallel and perpendicular to the uniform stream, respectively. The pitching moment coefficient was defined as

$$
C_{M}=-\frac{1}{H^{2}}\left[\int\left(X-\frac{1}{2} H\right) \Delta C p(X) \mathrm{d} X-\int Y \Delta C p(Y) \mathrm{d} Y\right]
$$

where $\Delta$ denotes the pressure coefficient difference at station $X$ or $Y$. In this research, it was decided to use the center of gravity of the base part as the center of pitching moment, because the aim of the experiment was to examine the effect of the stepped nose on the stability of the main body (base part).

The angle of attack $\alpha$ of the uniform stream to the obstacle was varied from $-30^{\circ}$ to $30^{\circ}$. In the case when the ob- stacle has non-zero angles of attack, the calculated forces were corrected by using the following equations:

$$
\begin{aligned}
& C_{D}=C_{D, \text { un }} \times \cos \alpha+C_{L, \text { un }} \times \sin \alpha \\
& C_{L}=C_{D \text {, un }} \times \sin \alpha+C_{L, \text { un }} \times \cos \alpha
\end{aligned}
$$

The force and moment coefficients calculated in this way are considered to have an accuracy on the order of 0.05 , because the back pressure fluctuates considerably.

The instrumentation for pressure and shedding frequency measurement were calibrated at various flow velocities. The calibration error for velocity measurement was within 1 percent. The error in setting angle of attack is estimated to be less than $0.5 \mathrm{deg}$, since the turntable at the test-section rotates manually. In the forces and moment measurement, an extra error occurs due to limitation in drilling the pressure orifice at the corners of the obstacle. However, this error is estimated to be less than 0.02 in non-dimensional value.

\section{Results and Discussion}

\subsection{Pressure measurement}

The variations of time-averaged surface pressure distribution for various step lengths and heights are shown in Fig. 2. The data obtained at fixed step height $h=0.16$ and at fixed step length $\ell=0.16$ are shown in Fig. 2(a) and Fig. 2(b), respectively. These data are selected in order to provide a comparison with the result of our previous numerical study. ${ }^{2)}$

Figure 2(a) indicates that the surface pressure distribution is significantly influenced by the step length. The abscissa, $s$, expresses the distance measured, in the counter clockwise (see Fig. 1), from the center of the obstacle's back surface along the perimeter of the obstacle's cross section toward the center of the front surface. It is normalized by the length of the base part. The change in step length affects not only the step region flow, but also the overall flow feature around the obstacle. The pressure coefficient at the center of the obstacle's back surface increases from -1.2 to -0.6 (the suction pressure acting on the back surface decreases) with increasing step length. The step length dependence of the pressure distributions on both sides of the base part $(0.5<$ $|s|<1.5)$ can be distinguished into two types. In the case of a short $\ell(\ell<0.16)$, the time-averaged pressure coefficient is almost unchanged along both side surfaces of the base part, implying that the flow is totally separated on both sides of the base part. On the other hand, in the case of a large $\ell(\ell \geq 0.16)$, the time-averaged pressure coefficient gradually increases downstream, implying that the flow attaches to the side surfaces of the base part. This change is consistent with the flow visualization result.

The change in pressure distribution due to step height variation (Fig. 2(b)) appears more drastic. The pressure coefficient on the back surface of the stepped-nose obstacle decreases from -0.6 to -1.8 . Observation of the pressure distribution on both side surfaces of the base part $( \pm 0.5<$ $s< \pm 1.5$ ) reveals that the dependence of side surface pressure coefficient on step height can also be distinguished into 


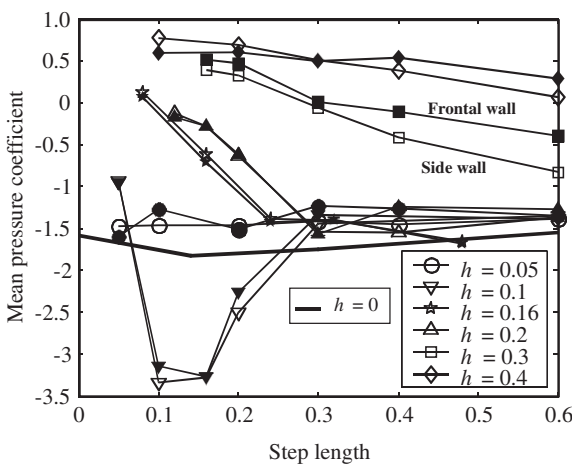

Fig. 3. Dependence of step region pressure on step length.

two types. In the case of a short $h(h \leq 0.16)$, the time-averaged pressure coefficient gradually increases downstream along either side surface of the base part, implying that the flow attaches to the side surfaces. In the case of a large $h(h>0.16)$, the time-averaged pressure coefficient is almost unchanged along both side surfaces of the base part. It is interesting to note that the pressure distributions for the cases of a short $\ell$ and a large $h$ are similar. In either case, the flow separating from the front surfaces impinges on the frontal step walls and then re-separates at the leading edges of the side surfaces of the base part.

Mean pressure coefficient along the step side walls (open symbols: $C p_{\text {ssw }}$ ) and frontal walls (solid symbols: $C p_{\text {sfw }}$ ) obtained at several step lengths and heights, are shown in Fig. 3. The effect of step length can be distinguished in three ways. First, in the case of a large step height $(h=0.3$ and 0.4 ), both $C p_{\text {ssw }}$, and $C p_{\text {sfw }}$ gradually decrease toward -1.25 . The flow visualization around the stepped-nose obstacle showed that the separated flow impinges on the step frontal wall and then re-separates at the leading edge of the side surface of the base part. In such a case, the step region pressure takes a high value and the thrust force created at the step walls becomes small. Second, in the case of $h=$ 0.2 and 0.16 , increasing $\ell$ until 0.3 causes $C p_{\text {ssw }}$, and $C p_{\text {sfw }}$ to gradually decrease. However, further increasing step length to $\ell=0.6$ causes no significant changes in $C p_{\text {ssw }}$, and $C p_{\text {sfw }}$. It is clear from flow visualization that the separated flow reattaches just at the leading edges of the base part side surfaces. Thus, the vortices trapped in the step regions are strengthened and the step region pressure decreases. Therefore, the thrust force created at the step walls maximizes. Third, in the case of $h=0.1$, increasing $\ell$ until 0.1 causes $C p_{\text {ssw }}$, and $C p_{\text {sfw }}$ to first decrease a little, then slightly increase when $\ell$ is increased to 0.16 . However, by further increasing $\ell$ to $0.3, C p_{\text {ssw }}$, and $C p_{\text {sfw }}$ drastically increase to -1.25 , and finally, increasing $\ell$ to 0.6 results in unchanged values of $C p_{\text {ssw }}$, and $C p_{\text {sfw }}$. Flow visualization reveals that the separated flow goes downstream without reattachment. Thus, the vortices trapped in the step regions are pulled out from the step regions, causing the increased step region pressure. Therefore, the thrust force created at the step walls becomes small.

Base pressure coefficient (open symbol: $C p b$ ) and mean

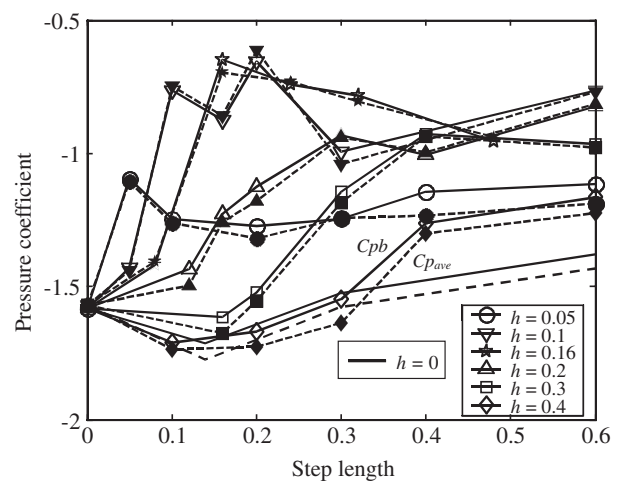

Fig. 4. Dependence of base pressure coefficient on step length.

back pressure coefficient (solid symbol: $C p_{\text {ave }}$ ) of the stepped-nose obstacle are related to the flow phenomena in the wake region. Figure 4 shows the variation of $C p b$ and $C p_{\text {ave }}$ for various $\ell$ and $h$ at zero angle of attack. It is found that the behavior of $C p b$ is almost identical to that of $C p_{\text {ave }}$. Therefore, the following discussion is based on the base pressure coefficient. The variation of $C p b$ with $\ell$ can be classified into three patterns. In the case of $h=0.4$, the negative base pressure gradually increases at $\ell>0.1$. Its slope is similar to that of a square obstacle. This implies that the $C p b$ characteristics are mostly determined by the flow pattern at the side surface of the obstacle, although the geometry of the stepped-nose obstacle, in the case of $h=0.4$, is quite different from the square obstacle. In the cases of $h=$ 0.3 and 0.2 , the negative $C p b$ rapidly increases with increasing $\ell$ until $\ell=0.4$. After that, the $C p b$ gradually increases with increasing $\ell$. It is obvious that increasing $\ell$ until 0.4 alters the whole flow pattern around the obstacle. In the cases of $h=0.1$ and 0.16 , the negative $C p b$ drastically increases to $C p b=-0.7$, fluctuates as $\ell$ is increased to $\ell=0.3$, and then increases gradually as $\ell$ is increased to $\ell=0.6$. It is observed that the effect of $\ell$ maximizes between $\ell=0.1$ to 0.2 , due to enhanced circulation in the step region, which avoid the flow separation on the side surface of base part.

\subsection{Drag coefficient}

Typically, the drag forces of blunt bodies at low angle of attack are larger than streamlined bodies, due to flow separation from both front corners. However, the stepped-nose obstacle could exhibit significant drag reduction. In the present investigation, we examined the drag of a stepped-nose obstacle for various $\ell$ and $h$ at a uniform stream velocity of $20 \mathrm{~m} / \mathrm{s}$. Figure 5 shows the contour of drag coefficient for various $\ell$ and $h$ for the case of a zero attack angle. The drag coefficient of a square cylinder $(\ell=0)$, measured in this experiment, is $C_{D}=2.26$, which agrees with previously reported values $\left(2.04\right.$ at $R e=1.76 \times 10^{5}$ in Lane and Loehrke experiment, ${ }^{6)} 2.15$ at $R e=1.3 \times 10^{4}$ in Norberg experiment, ${ }^{7)} 2.09$ at $1.0 \times 10^{5}$ in Murakami et al. $\mathrm{CFD}^{8,9)}$ ). Different behavior of drag coefficient of a stepped-nose obstacle is observed in two portions of the $h-\ell$ plots. Firstly, in the case of large step height and small step length, the flow separating from each front corner impinges on the step frontal wall. Although an increase in the step re- 


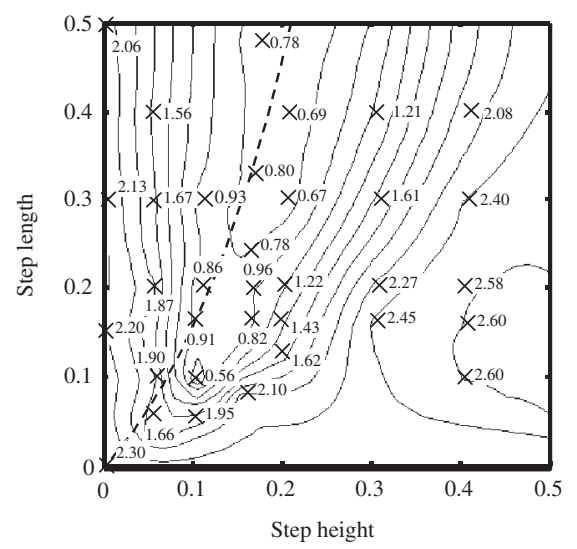

Fig. 5. Contour of drag coefficient for various step heights and step lengths.

gion pressure, caused by the impingement of the separated flow, tends to move the impinging point outward, the impinging point is still on the step wall due to a large step height. As a result, the drag coefficient of a stepped-nose obstacle in this case may be larger than that of a square or rectangular obstacle as seen in Fig. 5. Secondly, in the case of a relatively small step height and large step length, the flow separating from each front corner reattach at the leading edge of the side surface of the base part and significant drag reduction is attainable. The drag coefficient at the optimum step configuration reduces to less than half of the value of a square obstacle. Since the drag coefficient of a longer rectangular obstacle $(h=0)$ takes a smaller value, the measured drag coefficient of a stepped-nose obstacle includes the effect of obstacle length. The effect of the vortex trapping in the step region may identify with the difference between the drag coefficient of the stepped-nose obstacle $(h, \ell)$ under consideration and that of a rectangular obstacle $(h=$ $0, \ell)$. It is apparent from Fig. 5 that there is an optimum step configuration for drag reduction.

To understand the mechanism of large drag reduction at a certain step configuration, the contribution of each surface component of the stepped-nose obstacle is shown in Fig. 6(a). Since the step height is fixed at $h=0.16$ and the step length is varied, this figure characterizes the step length effects. The total drag coefficient reaches almost a constant value for $\ell>0.16$ that is dominated by the drag force acting on the back surface. This means that the thrust forces acting on the step walls cancel the drag force acting on the front surface. Figure 6(b) shows the contribution of each surface component for various $h$. The drag coefficient takes a minimum value for $h$ nearly equal to 0.16 . The forebody drag (the sum of the contributions of the front face and the step frontal walls) is almost zero at this step height. The figure indicates that the mechanism which brings about the reduction of the forebody drag, is the main cause in the reduction of total drag. That is, the drag reduction mechanism of the forebody changes the overall flow separation pattern and reduces the drag force acting on the back surface. Thus, it is concluded that the stepped-nose is effective to reduce the drag of the obstacle, when the net drag of the stepped-nose

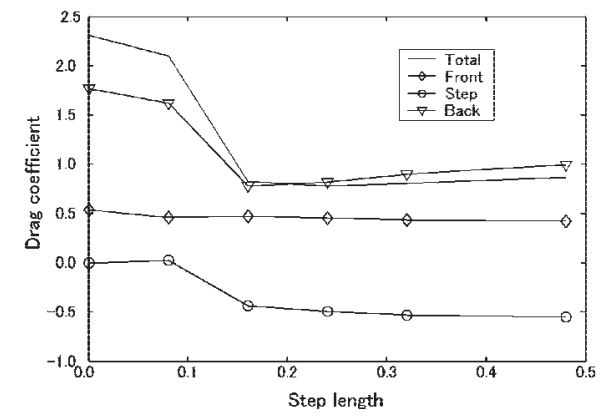

(a) Dependence of drag coefficient on step length

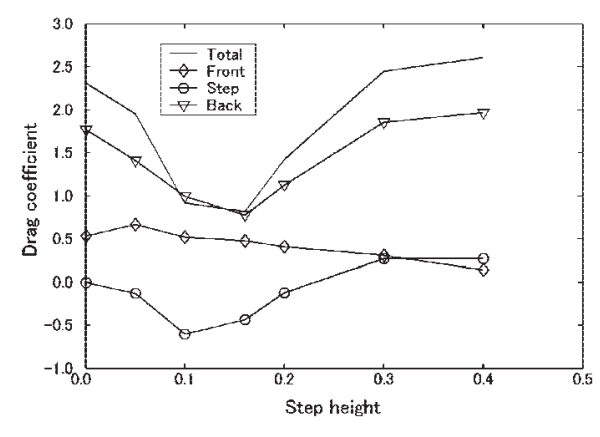

(b) Dependence of drag coefficient on step height

Fig. 6. Composition of drag coefficient.

part becomes zero, and the total drag acting on the obstacle is dominated by the drag acting on the back surface of the obstacle. In general, the step configuration, which realizes such a large drag reduction, can be identified from Fig. 5.

\subsection{Angle of attack effect}

The results of pressure measurement along the body surface of the stepped-nose obstacle, with $h=0.1$ and $\ell=0.1$, 0.16 and 0.2 for $\alpha=0$ to $28.5 \mathrm{deg}$, are shown in Fig. 7. As seen in Fig. 5, at $\alpha=0$, these stepped-nose obstacles have a large drag reduction effect. The result at the negative abscissa, $s$, which corresponds to the windward side, shows that increased angle of attack results in increased step region pressure because the flow separating from the front corner is pushed inward by the uniform stream and tends to impinge on the step wall. On the other hand, at positive $s$, which corresponds to the leeward side, increased angle of attack causes the total flow separation over the obstacle side, leading to a constant side pressure. These tendencies are commonly observed for any stepped-nose obstacle. However, in the case of $h=0.1$ and $\ell=0.16$ shown in Fig. 7(b), increasing the angle of attack from $\alpha=0$ to 3.5 results in the leeward step region pressure lower than the windward step region pressure. The pressure coefficient on the leeward step region decreases from -3.1 to -3.5 , while the pressure coefficient on the windward step region increase from -3.1 to -2.5 . This indicates that the separated flow in both step regions reattaches at the leading edges of the side surfaces of the base part. The differences between the two step region pressure coefficient is considered to be caused by the change in the inclination angle of the separated flow at the reattachment points, although more detailed measurement with better instrumentation, such as hot-film, is needed to support 


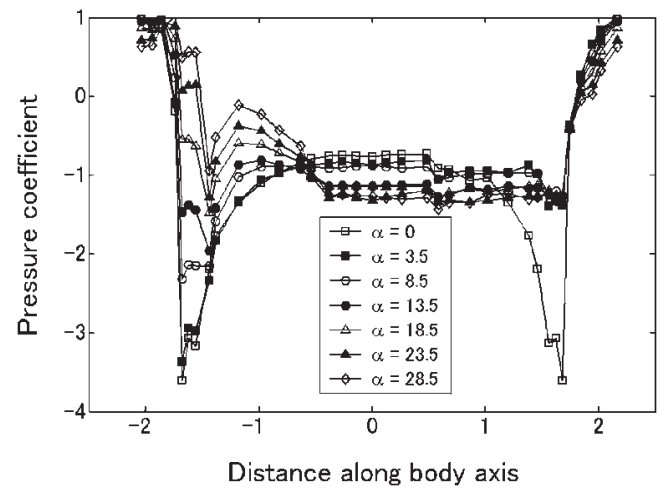

(a) Step length $=0.1$

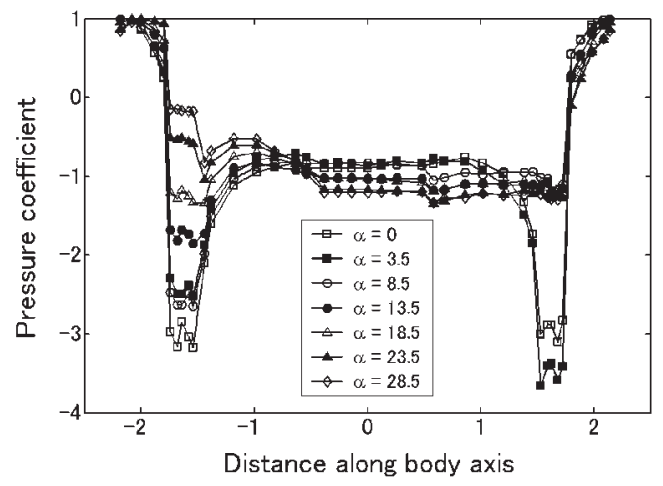

(b) Step length $=0.16$

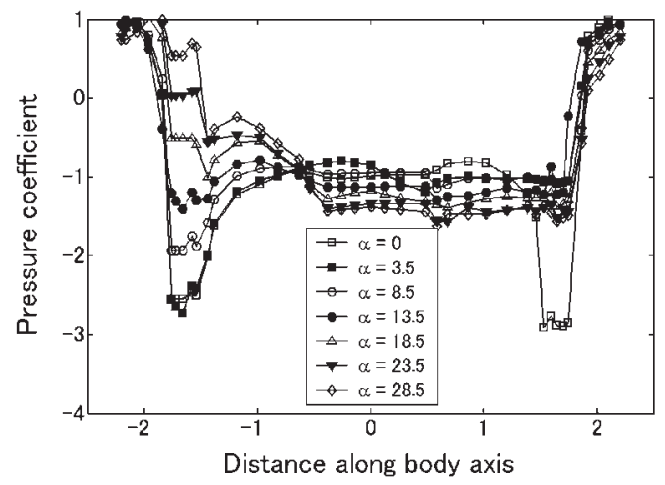

(c) Step length $=0.2$

Fig. 7. Surface pressure distribution for various angles of attack for fixed step height $h=0.1$.

this speculation.

The pressure acting on the back surface of a stepped-nose obstacle also varies with increasing angle of attack as shown in Fig. 7, where the base pressure is found to decrease with an increasing angle of attack, except at small angles of attack. It is observed that the base pressure coefficient of the stepped-nose obstacle has a similar value to the pressure coefficient on the side surfaces of the base part. This indicates that the flow separates without reattachment to the side surfaces the base part and creates a high suction pressure on the back surface of the obstacle.

Drag, lift and moment coefficients, which are calculated from the surface pressure distribution, are plotted against angle of attack for the cases of $h=0.1$ and $\ell=0.1,0.16$ and 0.2 in Fig. 8. It is interesting to examine the stability

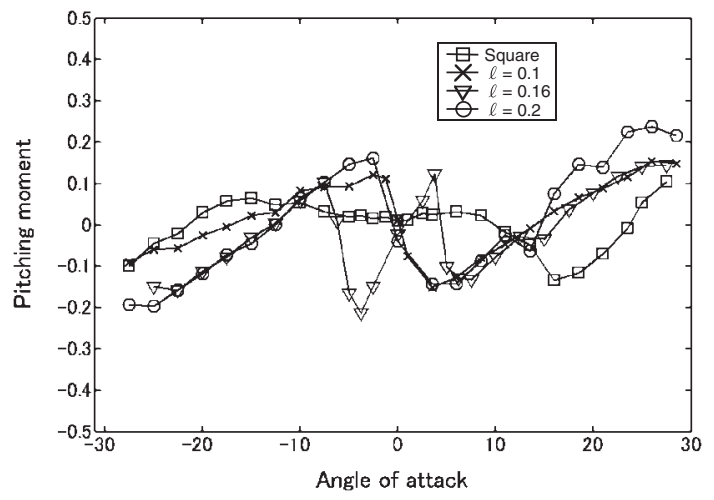

(a) Pitching moment coefficient variation

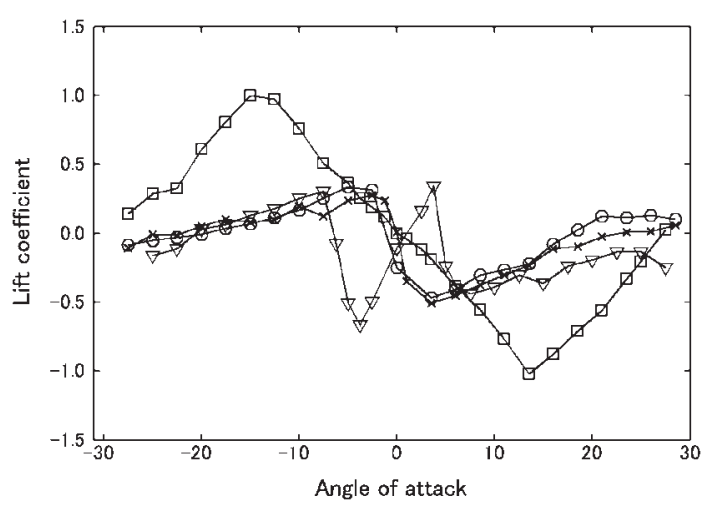

(b) Lift coefficient variation

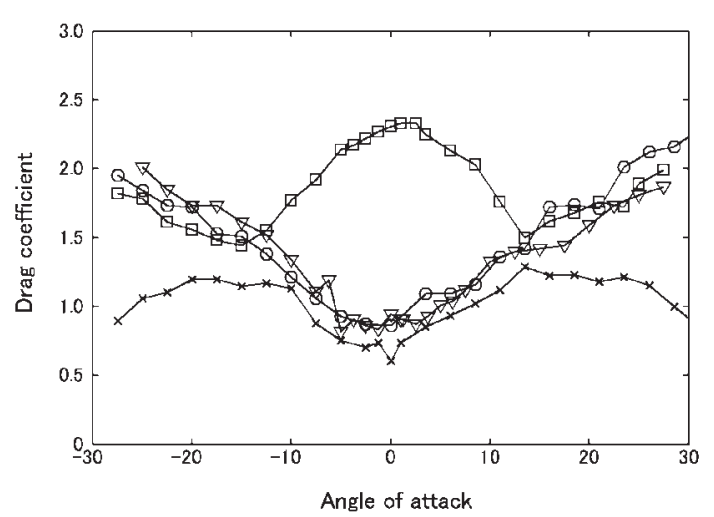

(c) Drag coefficient variation

Fig. 8. Drag, lift and moment coefficients plotted against angle of attack for fixed step height $h=0.1$ and various step lengths $\ell=0.1,0.16$ and 0.2 .

of a stepped-nose obstacle when the obstacle is free from external support. The obstacle is stable, has a tendency to return to stationary attitude after small disturbances. This condition is satisfied when, in Fig. 8(a) and (b), the slopes of the pitching moment coefficient is negative at zero angle of attack, and the slope of the lift coefficient is positive. Obviously, the square cylinder is unstable. Figure 8(a) and (b) indicate that the stepped-nose obstacles with $\ell=0.1$ and 0.2 are statically stable but dynamically unstable. The static stability emerges because, as seen in Fig. 8, the increased angle of attack from zero causes the decrease in the windward step region pressure but the increase in the leeward step region pressure. The underlying physics are the enhanced strength 
of the vortex trapped in the windward step region and the occurrence of total flow separation from the front corner in the leeward step region. For the same reason, the stepped-nose obstacles become dynamically unstable. However, a different behavior is observed for the stepped-nose obstacle with $\ell=0.16$, for which the increased angle of attack from zero causes the increase in both $C_{L}$ and $C_{M}$. This opposite behavior to other cases is observed in the range from $\alpha=-7.5$ to $\alpha=7.5$, where the flow pattern around the stepped-nose obstacle changes drastically. When the step region flow is in an optimal condition, the flow separating from the windward front corner is pushed inward to impinge on the windward step frontal wall. So that, the windward step region pressure can be still higher than the leeward step region pressure even if the latter is increased by the occurrence of total flow separation from the front corner.

The $C_{D}$-curves of $h=0.1$ plotted against angle of attack $\alpha$ for $\ell=0,0.1,0.16$ and 0.2 are shown in Fig. 8(c). The difference between the square obstacle $(\ell=0)$ and the stepped-nose obstacle is apparent in the range from $\alpha=-15$ to $\alpha=15$. The minimum drag coefficient is about 0.6 to 0.8 for the stepped-nose obstacle, which is obtained at zero angle of attack, and about 1.4 for the square obstacle, which is obtained at $\alpha=15$. At $\alpha= \pm 15$, both square and steppednose obstacle exhibit a similar flow pattern; the flow separates from the front surfaces without reattaching to the side surface. Thus, the effect of the stepped-nose is not effective. It is notable that, in the case $\ell=0.1$, the drag coefficient (denoted as cross symbol) is lower than the other cases for any angle of attack presented in Fig. 8(c).

\subsection{Strouhal number}

The measurement of vortex-shedding frequency was also conducted at zero angle of attack. The variation of the Strouhal number with $\ell$ for various $h$ is shown in Fig. 9 . The frequency of vortex shedding was determined by performing a Fast Fourier Transform (FFT) on the velocity fluctuation, which was measured by using a single hotwire at a distance $4 H$ from the back surface of the stepped-nose obstacle. The Strouhal number is defined by $S t=f H / U_{\infty}$, where $f$ is the shedding frequency, $H$ denotes the obstacle height and $U_{\infty}$ is the uniform stream velocity which is as-

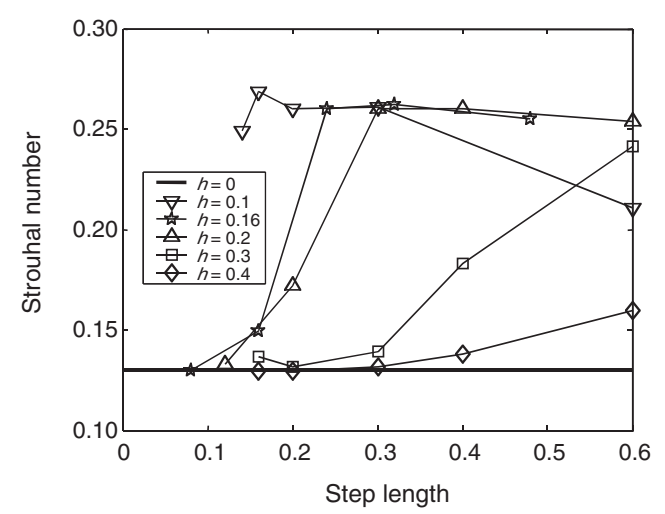

Fig. 9. Dependence of Strouhal number on step length for various step heights. sumed to be constant. In this investigation, we used several rectangular obstacles for measuring the wake-shedding frequency of $h=0$-case. The spectrum for the square/rectangular obstacle, denoted by the thick solid line in Fig. 9, yields a constant Strouhal number $(S t=0.13)$. This implies that the wake flow does not significantly change with increasing the obstacle's length alone so far as the obstacle's length is relatively short. This result is consistent with Okajima's experiment ${ }^{10)}$ which shows that the Strouhal numbers of a square cylinder, and rectangular cylinders with width to height ratio being equal to 2 and 3 at a Reynolds number close to $1 \times 10^{4}$ are similar. For the square cylinder (step length $\ell=0$ ), flow separation at each front corner causes a circulation region on either side surface of the obstacle because the separated flow goes downstream without reattachment. Note that, in the case of $h=0.4$, the Strouhal number slowly increases with increasing step length. In particular, for the step length less than $\ell=0.3$, the Strouhal number of the stepped-nose obstacle takes the same value as the square/rectangular obstacle case. This is because the re-separation of flow at the leading edge of the side surfaces of the base part causes large scale separation flow on both sides of the stepped-nose obstacle and the overall flow pattern around the base part is similar with to that of a square.

On the other hand, for the stepped-nose obstacle with step height $h=0.1,0.16$ and 0.2, the Strouhal number drastically increases with increasing step length. This change occurs because the reattachment of flow to both sides of the base part makes the width of wake flow narrower. The maximum Strouhal number measured in the experiment is $S t=0.27$, which is higher than the Strouhal number of a circular cylinder. The transitional changes in the Strouhal number of the stepped-nose obstacle, observed in Fig. 9, correspond to the occurrence and suppression of the flow separation on both side surfaces of the base part.

Figure 10 shows the measurement results on the steppednose obstacle with $h=0.1$ and various $\ell$ for the variations of the base pressure, drag coefficient and Strouhal number plotted against angle of attack. It is clear that the base pressure and Strouhal number of stepped-nose obstacle has a reciprocal correlation to the drag coefficient. It is also confirmed that the stepped-nose obstacle with small size of step $(\ell=0.05)$ behaves similarly to the square obstacle.

\section{Conclusions}

The effects of a stepped-nose with various step lengths and heights have been experimentally investigated. It was found that there is an optimum step configuration which makes the net drag force acting on the forebody to vanish with stabilized side flow on both sides of the square form of main body.

The pressure measurement was conducted for various step lengths $(0<\ell<1)$ and step heights $(0<h<0.5)$. It was observed that the time-averaged pressure coefficient distribution in the case of short $h(h<0.16)$ gradually increases downstream along either side surface of stepped ob- 


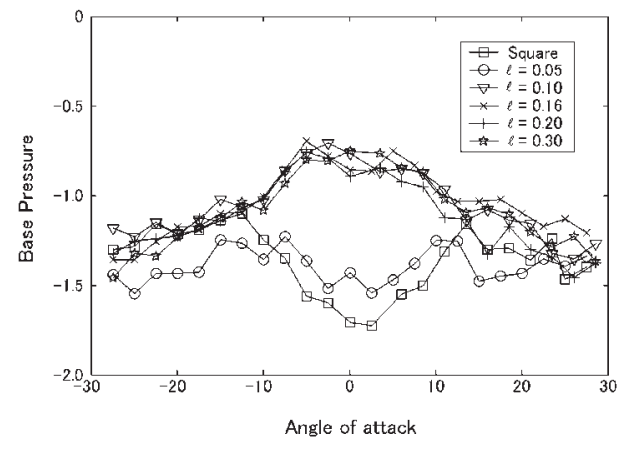

(a) Base pressure variation

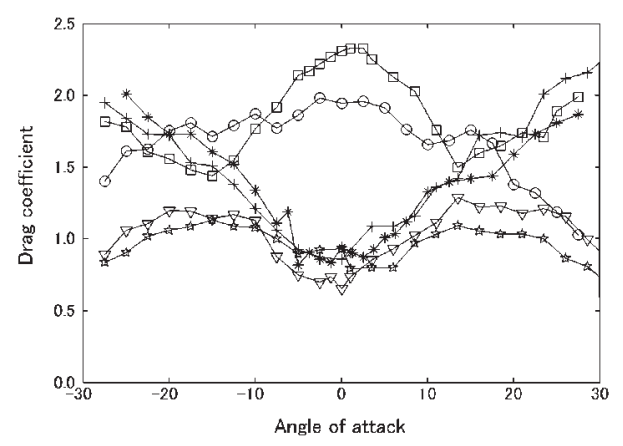

(b) Drag coefficient variation

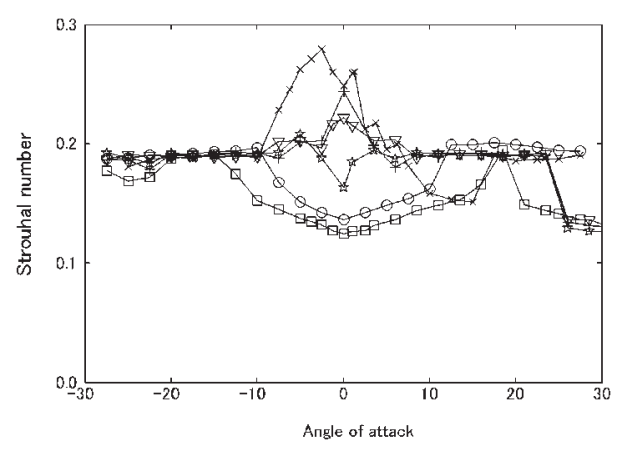

(c) Strouhal number variation

Fig. 10. Dependence of base pressure, drag coefficient, and Strouhal number on angle of attack for fixed $h=0.1$.

stacle, implying that the flow attaches to the side surfaces. In the case of large $h(h>0.16)$, the time-averaged surface pressure coefficient is almost unchanged along both side surfaces of the base part.

The maximum thrust force acting on the frontal step walls was observed in the case of $h=0.1$, where the step region pressure drastically decreases due to the vortex trapped in the step region and the acceleration of the separated flow. On the other hand, the force acting on the frontal step walls changed to the drag force in the case of $h=0.4$, where the pressure acting on the step frontal wall increases due to the impingement of separated flow on the step's frontal wall.

We have examined the drag coefficient of a stepped-nose obstacle for various $\ell$ and $h$ at a uniform stream velocity of $20 \mathrm{~m} / \mathrm{s}$. In the case of a large $h$ and small $\ell$, the flow sepa- rating from the front corners impinges on the step walls. Since the separated flow cannot fully be accelerated by the circulation flow in the step region, the step region pressure takes a positive value and the drag coefficient of the stepped-nose obstacle becomes larger than that of simplesquare or rectangular obstacles. Significant drag reduction occurs in the case of relatively small $h$ and large $\ell$. In particular, the maximum drag reduction is attained in the case of $h=0.1$ and $\ell=0.16$ for which the flow separating from the front corners attaches smoothly to the leading edges of the base side surface. The drag of stepped-nose obstacle is more sensitive to the step height change then the step length change.

The surface pressure measurement results along the body surface of the stepped-nose obstacle for $h=0.1$ and $\ell=$ $0.1,0.16$ and 0.2 at $\alpha=0$ to $30 \mathrm{deg}$ show the total flow separation on the windward side of the obstacle while the leeward step region flow keeps the optimum flow configuration. It is concluded that the step region pressures determines the characteristics of the side surface and the base pressures. These contribute to $C_{L}, C_{D}$, and $C m$ significantly, resulting in stable the stepped-nose obstacle attitude.

For the stepped-nose obstacle, with stepped height $\ell=$ $0.1,0.16$ and 0.2 , the Strouhal number drastically increases, with increasing step length. The maximum Strouhal number measured in the experiment is $S t=0.27$. The stepped-nose obstacle with large drag reduction has a higher Strouhal number than a circular cylinder.

\section{References}

1) Watanabe, K.: Characteristics of Axial Flow around Step Cylinder (Parallel Flow), Trans. Jpn. Soc. Mech. Eng. B, 62 (1996), pp. 2130-2136 (in Japanese).

2) Cakrawala, A. and Umemura, A.: Drag Reduction and Flow Stabilization Mechanism of Step Nose Obstacle, Proceeding of the 39th Aircraft Symposium, 2001.

3) Cakrawala, A. and Umemura, A.: An Experimental on Turbulent Measurement and Side Flow by Using Stepped-Nose Obstacle, Proceeding of the 40th Aircraft Symposium, 2002.

4) Koenig, K. and Roshko, A.: An Experimental Study of Geometrical Effects on the Drag and Flow Field of Two Bluff Bodies Separated by a Gap, J. Fluid Mech., 156 (1985), pp. 167-204.

5) Ruderich, R. and Fernholz, H. H: An Experimental Investigation of a Turbulent Shear Flow with Separation, Reverse Flow, and Reattachment, J. Fluid Mech., 163 (1986), pp. 283-322.

6) Lane, J. C. and Loehrke, R. I.: Leading Edge Separation from Blunt Plate at Low Reynolds Number, Trans. ASME, 102 (1980), pp. 494496.

7) Norberg, C.: Flow around Rectangular Cylinder: Pressure Force and Wake Frequencies, J. Wind Eng. Ind. Aerodyn., 49 (1993), pp. 187196.

8) Murakami, S., Mochida, A. and Sakamoto, S.: CFD Analysis of WindStructure Interaction for Oscillating Square Cylinder, J. Wind Eng. Ind. Aerodynam., 72 (1997), pp. 33-46.

9) Murakami, S. and Mochida, A.: On Turbulent Vortex Shedding Flow Past 2D Square Cylinder Predicted by CFD, J. Wind Eng. Ind. Aerodynam., 54/55 (1995), pp. 191-211.

10) Okajima, A.: Strouhal Numbers of Rectangular Cylinder, J. Fluid Mech., 123 (1982), pp. 379-398. 